\title{
A Systematic Review of Asynchronous, Provider-to-Provider, Electronic Consultation Services to Improve Access to Specialty Care Available Worldwide
}

Clare Liddy, MD, MSc, CCFP, FCFP, ${ }^{1,2}$ Isabella Moroz, PhD, ${ }^{1}$

Ariana Mihan, BSC, ${ }^{1}$ Nikhat Nawar, ${ }^{3}$ and Erin Keely, MD, FRCPC ${ }^{3,4}$

${ }^{1}$ C.T. Lamont Primary Health Care Research Center, Bruyère Research Institute, Ottawa, Canada.

${ }^{2}$ Department of Family Medicine, University of Ottawa, Ottawa, Canada.

${ }^{3}$ Department of Medicine, University of Ottawa, Ottawa, Canada.

${ }^{4}$ Division of Endocrinology/Metabolism, The Ottawa Hospital, Ottawa, Canada.

\section{Abstract}

Background: Electronic consultation (eConsult) is an asynchronous electronic communication tool allowing primary care providers to obtain a specialist consultant's expert opinion in a timely manner, thereby offering a potential solution to excessive wait times for specialist care, which remain a serious concern in many countries.

Introduction: Our 2014 review of eConsult services demonstrated feasibility and high acceptability among patients and providers. However, gaps remain in knowledge regarding eConsult's impact on system costs and patient outcomes.

Materials and Methods: Following the PRISMA guidelines, we conducted a systematic review in May 2017 of English and French literature on OVID Medline, EMBASE, ERIC, and CINAHL databases, examining all studies on eConsult services published since our previous review. The Quadruple Aim Framework was used to synthesize outcomes. Articles reporting on the impact of eConsult on access, patient safety and satisfaction, utilization rates, clinical workflow, and continuing medical education were analyzed using a narrative synthesis approach.

Results: The initial search yielded 1,021 results, 50 of which were included on abstract and received a quality assessment and full text review. Of these, 43 were included in our final analysis. Results demonstrated the worldwide presence of eConsult services in North America and countries beyond, including Brazil, Australia, Spain, and The Netherlands. The breadth of specialty services offered has greatly expanded beyond dermatology and includes cardiology, nephrology, and hematology among others.
Overall impact on access measures, acceptability, cost, and provider satisfaction remain positive. There is limited research on population health outcomes of morbidity and mortality.

Conclusions: The availability of eConsult services has spread both geographically and in terms of specialty services offered. By allowing for a greater population to be served, access to care is being improved; however, long-term impact should continue to be assessed with a focus on patient safety, morbidity, mortality, and cost effectiveness metrics.

Keywords: eConsult, telehealth, e-health, telemedicine, technology

\section{Introduction}

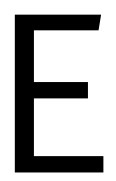

lectronic consultation (eConsult) is an emerging health innovation designed to address excess wait times for specialist care by enabling primary care providers (PCPs) to obtain a specialist consultant's expert opinion in a timely manner. According to a 2016 Commonwealth Fund report, wait times for specialist care in Canada were the highest out of 11 countries surveyed. ${ }^{1}$ In Canada, 56\% of patients waited at least 4 weeks to see a specialist, a trend that has remained consistent since 2010 and is significantly higher than the Commonwealth Fund average of 36\%. Furthermore, results of the report show that one in five Canadians report that their PCP is not informed about their specialist visit, indicating a need for improved communication between PCPs and specialists similar to the international average. ${ }^{1}$

Other countries face similar challenges in accessing specialist advice. A recent wait time report in England showed that in the first 7 months of 2017, 89.4\% of patients waited up to 18 weeks to see a specialist. This result failed to meet their standard of $92 \%$ of patients waiting no more than 18 weeks for their consult. ${ }^{2}$ In Northern Ireland, statistics from the first 6 months of 2017 revealed that $71.6 \%$ of patients waited longer than 9 weeks to see a specialist and $24.2 \%$ waited more than 52 weeks. These results have increased from $64.8 \%$ and $13.4 \%$, respectively, in the same timeframe of $2016 .^{3}$ In Brazil, 


\section{SYSTEMATIC REVIEW OF ECONSULT SERVICES}

specialists described the referral process as unorganized, lacking patient triaging, and consisting of inadequate communication between providers. ${ }^{4}$

Previous reviews evaluating Consult's use and impact demonstrated its overall feasibility in application and its ability to improve timely access to specialty care. ${ }^{5,6}$ However, both studies revealed a lack of literature analyzing the cost effectiveness of eConsult and its impact on patient outcomes and safety. It was additionally shown that many eConsult services were primarily limited to single specialty services, particularly dermatology, thus generalizability of impact across other specialty areas was not established.

Therefore, we conducted a systematic review to expand on past reviews of the literature and examine eConsult's impact on delivery of care by applying the Quadruple Aim Framework. ${ }^{7}$ This framework proposes that the optimization of the performance of the health system should involve simultaneously pursuing four interdependent objectives: population health, experience of receiving care (patients), experience of providing care (providers), and per capita costs. This review will provide value in informing recently published perspectives, developments, and implications of eConsult services worldwide.

\section{Methods}

\section{STUDY DESIGN}

This is a systematic review analyzing the impact of eConsult on the delivery of care. The protocol was registered on PROSPERO.2017, CRD42017069139.

\section{DATA SOURCES}

On May 11, 2017 we conducted a search through OVID of Medline (from 1946), EMBASE (from 1947), and ERIC (1965 to April 2017) and through EBSCO of CINAHL. Titles published from August 2014 to May 2017 in English or French were included. We expanded on our previous review by including ERIC and CINAHL in our search databases. The search strategy consisted of two search clusters of keywords: (1) keywords for electronic consultation (e.g., eConsult*, teleconsult*) and (2) keywords for provider care (e.g., general practitioner [GP], specialist care). Our previous review only focused on terms regarding eConsult and primary care. We broadened the second search cluster to include search terms regarding both primary and specialty care.

\section{INCLUSION CRITERIA}

Records were screened for publication type and relevance to eConsult. Conference proceedings/articles/abstracts, editorials, letters, notes, reviews and opinion pieces, policy papers, guidelines, pilot, and case studies were excluded. Distiller SR was used to screen titles and abstracts based on predetermined relevancy criteria. eConsult was defined as an asynchronous, directed communication between providers over a secure electronic medium that involved sharing of patient-specific information and sought clarification or guidance regarding clinical care. This excluded interventions, such as web-based discussion forums, e-mail communication (unless explicitly described as secure), videoconferencing, one-way communications, and services connecting patients and providers. Examples of PCPs included family doctors and nurse practitioners. Articles that defined dentists and pharmacists as PCPs were excluded.

Two reviewers (A.M. and N.N.) screened the abstracts for relevancy, with a third reviewer (I.M.) verifying 15 of the screened articles for rigor. C.L. and I.M. performed a second abstract screen to further eliminate (or include) any articles for full screening. Full articles were then screened for relevancy by N.N. and A.M.

A modified version of the Effective Public Health Practice Project (EPHPP) Quality Assessment Tool for Quantitative Studies by Thomas et al. ${ }^{8}$ was used to assess the quality of articles. $^{5}$ The review team consisted of 4 members (C.L., E.K., I.M., and A.M.), each assessing 11-12 articles.

\section{THE QUADRUPLE AIM FRAMEWORK}

In 2008, Berwick et al. first described the Triple Aim Framework as simultaneously improving population health, improving the patient experience of care, and reducing per capita cost. ${ }^{9}$ The Quadruple Aim Framework, proposed in 2014 by Bodenheimer and Sinksy, builds on Berwick et al.'s work by introducing a fourth dimension, provider experience of care, which researchers have described as an essential component to adoption and sustainability. ${ }^{7}$ Therefore, the updated framework, which we have used in the present review (Table 1), includes the provider experience dimension and the corresponding outcome measures.

\section{DATA EXTRACTION}

We followed the data extraction template used in our previous review with a few modifications to capture the dimensions of the Quadruple Aim Framework. ${ }^{7}$ The potential impacts and outcome measures are shown in Table 1. Items extracted included title, author, location, research question/ objective, study design, service type, sample size, main outcomes, and findings.

\section{ANALYSIS}

Using the Quadruple Aim Framework as a guide, we conducted a narrative synthesis of included studies. While the articles included in the study use a variety of terms to describe 


\section{LIDDY ET AL.}

\begin{tabular}{|c|c|c|}
\hline Population Health & $\begin{array}{l}\text { Population-level outcomes with a defined denominator (e.g. } \\
\text { chronic pain patients, patients in rural regions, etc.) }\end{array}$ & $\begin{array}{l}\text { Traditional population health outcomes: mortality, health and } \\
\text { functional status, healthy life expectancy } \\
\text { Disease burden (e.g., incidence and/or prevalence rates } \\
\text { of major chronic conditions) } \\
\text { Behavioral and physiological factors (e.g., a composite health } \\
\text { risk assessment score) }\end{array}$ \\
\hline Experience of care (patient) & Patient-reported outcome and experience measures & $\begin{array}{l}\text { Standard questions from patient surveys (e.g., health status, } \\
\text { quality of life) } \\
\text { Set of measures based on key dimensions (e.g., Institute of } \\
\text { Medicine's six aims for improvement: safe, effective, timely, } \\
\text { efficient, equitable, and patient centered) }\end{array}$ \\
\hline Per capita cost & $\begin{array}{l}\text { Downstream healthcare utilization costs, impact } \\
\text { of delayed medical referral }\end{array}$ & $\begin{array}{l}\text { Hospital and ED utilization rates and costs } \\
\text { Other contacts with healthcare services (e.g., tests, specialist } \\
\text { consultations) and associated costs }\end{array}$ \\
\hline
\end{tabular}

$E D$, emergency department.

their electronic consultation services (e.g., e-consultation, teleconsultation), for the purposes of this study we will use the term eConsult exclusively.

\section{Results}

A search of target databases on May 11, 2017 revealed 1,021 citations. A total of 43 studies were used for data extraction after limiting publication dates to 2014-present, excluding articles not published in English or French, and removing duplicates and ineligible articles (Fig. 1).

Table 2 shows that the majority of studies were conducted in the United States (44\%) and Canada (28\%), with some in Brazil (9\%), Europe (Spain, Italy, Austria, The Netherlands; 7\%), and Australia (2\%). In addition, two services extend globally across countries: Médecins Sans Frontières (MSF) and Naval Medical Center Portsmouth (NMCP) for military services around the world.

The breadth of specialty services offered has greatly expanded beyond dermatology, with most studies focused on multispecialty service (84\%), ranging from 8 to 86 specialties. Only $7(16 \%)$ studies described a single specialty service, which included services in the following specialty groups: dermatology, cardiology, and pulmonology. Figure 2 shows the distribution of top specialties accessed through eConsult, with endocrinology being the most frequently accessed at $19 \%$, followed by dermatology (16\%), hematology (9\%), cardiology (9\%), gastroenterology 8\%), and neurology (7\%). The number of eConsults reported ranged from 65 to 217,014, and number of participating providers ranged from 17 to 4,260 PCPs and 4 to 479 specialists. Most articles described eConsult for PCP-specialist communication, with only one reporting on specialist-to-specialist communication. ${ }^{10}$

Study designs varied with most studies (40\%) classified as retrospective and cross-sectional (16\%), and smaller proportion of mixed method designs (7\%) and randomized controlled trials (RCTs; 7\%). Due to the fact that most studies were descriptive in nature, the majority (88\%) of articles were categorized as "weak" based on the quality assessment tool. We did not exclude any articles based on the quality assessment.

There were multiple articles describing six eConsult services, which accounted for 67\% $(n=29)$ of the articles: 30\% $(n=13)$ from the Champlain BASE ${ }^{\mathrm{TM}}$ eConsult service, 9\% $(n=4)$ from the TNMG (Telehealth Network of Minas Gerais) in Brazil, 7\% $(n=3)$ from the Mayo Clinic, 7\% $(n=3)$ from San Francisco General Hospital, 5\% $(n=2)$ from the University of California San Francisco (UCSF), 5\% $(n=2)$ from MSF, and 5\% $(n=2)$ were from the Veterans Health Administration (VHA) (Table 3).

eConsult services were implemented on a variety of technological platforms (Table 4). Several services, including those at the VHA, ${ }^{11,12}$ Veteran's Affairs (VA),${ }^{13-15}$ UCSF, ${ }^{16,17}$ and the Mayo Clinic, ${ }^{10,18,19}$ use private networks, where the eConsult service is embedded into an electronic health record shared by referring providers and specialists, enabling the 


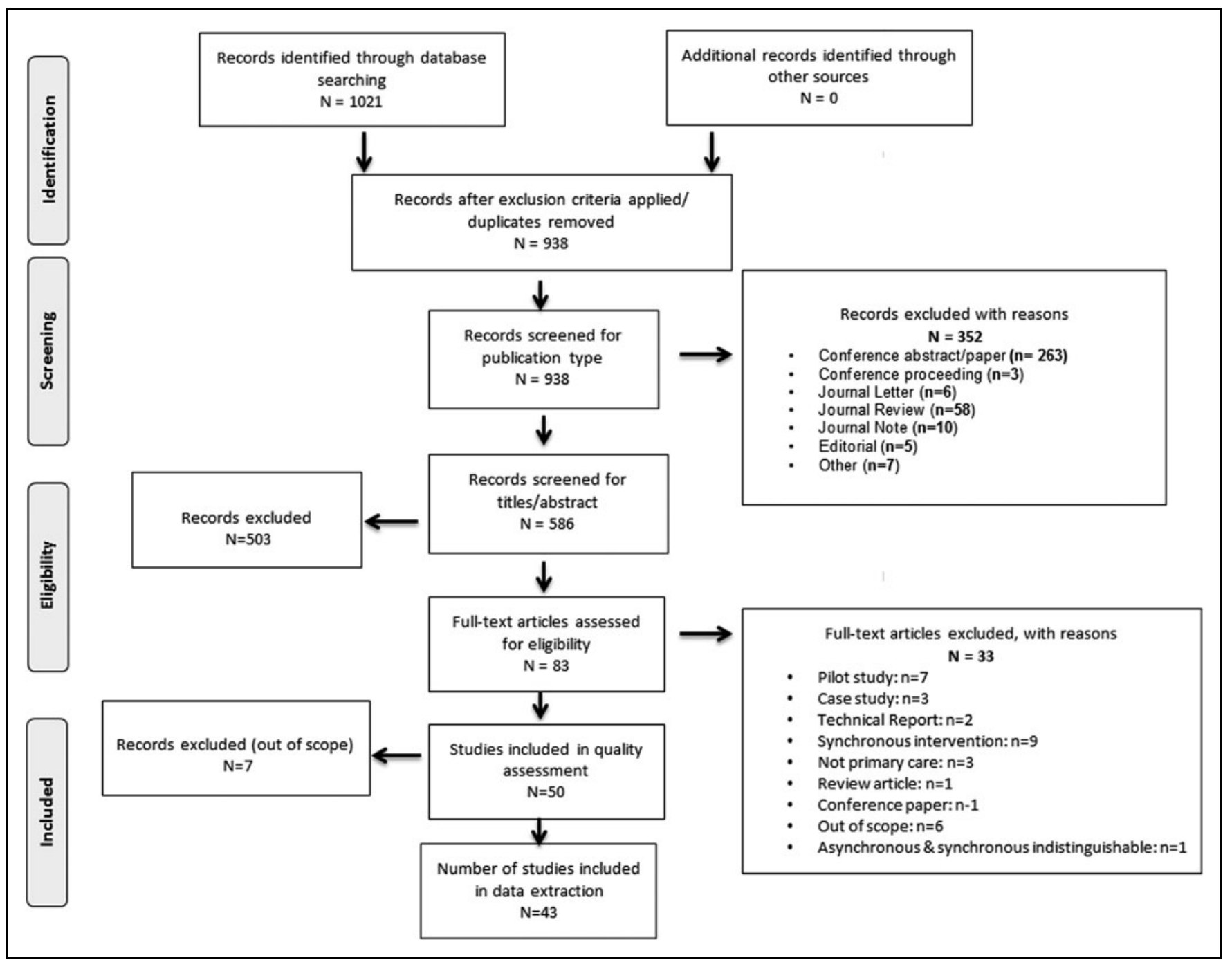

Fig. 1. Flow chart for study inclusion.

specialist to directly access patient information. Other eConsult services are independent of individual health records and use a secure web-based portals to link providers, such as the Champlain BASE in Canada, ${ }^{20-32}$ TNMG in Brazil, ${ }^{33-36}$ and KSYOS in The Netherlands, ${ }^{37}$ or harness secure intranet e-mail as the means of communication, such as Army Knowledge Online (AKO). ${ }^{38}$

\section{POPULATION HEALTH OUTCOMES}

There were very few reports of eConsult's impact on traditional population health outcomes, such as risk and mortality. In a RCT of cardiology eConsults, patient outcomes, including adverse events (e.g., death, myocardial infarction, coronary artery bypass grafting, urgent or emergent cardiac catheterization and/or angioplasty) as well as hospital and emergency department utilization for potential cardiac complaints or events, were compared between those patients who had an eConsult versus those referred through a traditional referral pathway. ${ }^{39}$ The results showed that those who had an eConsult were significantly more likely (1.5 times) to receive a consultation with a cardiologist and had lower rates of emergency department utilization than those who had traditional face-toface consultations. ${ }^{39}$ In another study, Price et al. ${ }^{40}$ conducted an assessment of patient harm based on a review of all electronic referrals to a gastroenterology clinic that did not result in scheduling of appointment. Within referrals considered resolved (where the referral complaints have been resolved or addressed), only nine patients (9\%) received unplanned care while awaiting scheduling decisions, five of whom experienced harm that was related to referral complaints, although 


\begin{tabular}{|c|c|c|c|c|c|c|c|}
\hline $\begin{array}{c}\text { FIRST } \\
\text { AUTHOR }\end{array}$ & YEAR & SETTING & $\begin{array}{l}\text { TYPE OF } \\
\text { SERVICE }\end{array}$ & $\begin{array}{l}\text { STUDY } \\
\text { DESIGN }\end{array}$ & $\begin{array}{c}\text { STUDY } \\
\text { POPULATION }\end{array}$ & OUTCOMES & $\begin{array}{l}\text { QUALITY } \\
\text { RATING }\end{array}$ \\
\hline Alkmim $^{35}$ & 2015 & Brazil & Multispecialty & $\begin{array}{l}\text { Randomized } \\
\text { qualitative study }\end{array}$ & 640 Teleconsultations & $\begin{array}{l}\text { Objectivity, courtesy, } \\
\text { ethics, quality }\end{array}$ & Weak \\
\hline Alkmim $^{34}$ & 2015 & Brazil & Multispecialty & Cross-sectional & $\begin{array}{l}1,954 \\
\text { Teleconsultations }\end{array}$ & $\begin{array}{l}\text { Factors associated with } \\
\text { teleconsultation utilization } \\
\text { rates }\end{array}$ & Weak \\
\hline Barnett $^{44}$ & 2017 & United States & Multispecialty & $\begin{array}{l}\text { Retrospective } \\
\text { observational } \\
\text { analysis }\end{array}$ & 3,060 PCPs & $\begin{array}{l}\text { Access, patterns of use, } \\
\text { response time, } \\
\text { sustainability }\end{array}$ & Weak \\
\hline Bonnardot ${ }^{45}$ & 2014 & Global (MSF) & Multispecialty & $\begin{array}{l}\text { Retrospective } \\
\text { analysis }\end{array}$ & $\begin{array}{l}\text { 1,039 Telemedicine } \\
\text { cases, } 163 \text { providers }\end{array}$ & $\begin{array}{l}\text { Response time, provider } \\
\text { satisfaction, education, } \\
\text { improved patient } \\
\text { management }\end{array}$ & Weak \\
\hline Byrom $^{42}$ & 2016 & Australia & Single specialty & $\begin{array}{l}\text { Retrospective case } \\
\text { analysis }\end{array}$ & 406 Telederm cases & $\begin{array}{l}\text { Response time, } \\
\text { concordance rate, face-to- } \\
\text { face recommendations }\end{array}$ & Weak \\
\hline $\mathrm{Cruz}^{17}$ & 2015 & United States & Multispecialty & Program evaluation & 158 eConsults & $\begin{array}{l}\text { Response time changes in } \\
\text { wait time), Downstream } \\
\text { utilizations (ED visits, } \\
\text { hospitalizations, and } \\
\text { specialty clinic visits), PCP } \\
\text { compliance with specialist } \\
\text { recommendations }\end{array}$ & Weak \\
\hline Datta $^{14}$ & 2015 & United States & Single specialty & $\begin{array}{l}\text { Randomized clinical } \\
\text { trial }\end{array}$ & $\begin{array}{l}196 \text { Patients } \\
\text { (conventional), } 195 \\
\text { patients } \\
\text { (teledermatology) }\end{array}$ & $\begin{array}{l}\text { Total and per participant } \\
\text { costs, effectiveness (trade } \\
\text { off utility) }\end{array}$ & Weak \\
\hline Delaigue $^{41}$ & 2014 & $\begin{array}{l}\text { Multicountry (Sudan, } \\
\text { Ethiopia, Congo) }\end{array}$ & Single specialty & $\begin{array}{l}\text { Retrospective } \\
\text { analysis utilizing } \\
\text { mixed methods }\end{array}$ & $\begin{array}{l}65 \text { Teledermatology } \\
\text { cases }\end{array}$ & $\begin{array}{l}\text { Response time, areas for } \\
\text { improvement of system, } \\
\text { quality of clinical details }\end{array}$ & Weak \\
\hline Fogel $^{20}$ & 2016 & Canada & Multispecialty & Cross-sectional & $\begin{array}{l}436 \text { Hematology } \\
\text { eConsults submitted } \\
\text { by } 171 \text { PCPs }\end{array}$ & $\begin{array}{l}\text { Avoidance of referrals, } \\
\text { common question types }\end{array}$ & Weak \\
\hline Fort ${ }^{52}$ & 2017 & United States & Multispecialty & Mixed methods & $\begin{array}{l}10 \text { Patients, } 18 \text { safety } \\
\text { net clinicians, } 12 \\
\text { specialists, } 3 \text { referral } \\
\text { coordinators, } 602 \\
\text { eConsults }\end{array}$ & $\begin{array}{l}\text { Patient satisfaction, } \\
\text { provider satisfaction }\end{array}$ & Weak \\
\hline Golberstein $^{48}$ & 2017 & United States & Multispecialty & $\begin{array}{l}\text { Cluster-randomized } \\
\text { evaluation }\end{array}$ & $\begin{array}{l}72 \text { Control PCPs, } 70 \\
\text { Treatment (eConsult) } \\
\text { PCPs }\end{array}$ & $\begin{array}{l}\text { Effect of eConsult on PCP } \\
\text { perception and their } \\
\text { ability to provide care for } \\
\text { mental health services }\end{array}$ & Weak \\
\hline Gupte $^{15}$ & 2016 & United States & Multispecialty & $\begin{array}{l}\text { Observational quality } \\
\text { improvement study }\end{array}$ & 7,097 eConsults & $\begin{array}{l}\text { Provider experience, } \\
\text { response type, most } \\
\text { frequent specialty } \\
\text { consulted }\end{array}$ & Weak \\
\hline Johnston ${ }^{25}$ & 2017 & Canada & Multispecialty & Cross-sectional & 85 eConsults & $\begin{array}{l}\text { Provider satisfaction, } \\
\text { topics of interest and } \\
\text { efficiency of eConsult in } \\
\text { pediatric hematology/ } \\
\text { oncology }\end{array}$ & Weak \\
\hline
\end{tabular}




\begin{tabular}{|c|c|c|c|c|c|c|c|}
\hline $\begin{array}{c}\text { FIRST } \\
\text { AUTHOR }\end{array}$ & YEAR & SETTING & $\begin{array}{l}\text { TYPE OF } \\
\text { SERVICE }\end{array}$ & $\begin{array}{l}\text { STUDY } \\
\text { DESIGN }\end{array}$ & $\begin{array}{c}\text { STUDY } \\
\text { POPULATION }\end{array}$ & OUTCOMES & $\begin{array}{l}\text { QUALITY } \\
\text { RATING }\end{array}$ \\
\hline Keely ${ }^{30}$ & 2015 & Canada & Multispecialty & Survey & 34 Specialists & $\begin{array}{l}\text { Provider experience/ } \\
\text { satisfaction }\end{array}$ & Weak \\
\hline Kirsh $^{12}$ & 2015 & United States & Multispecialty & Observational cohort & 217,014 eConsults & $\begin{array}{l}\text { Growth of eConsults by } \\
\text { VHA regional networks, } \\
\text { medical centers and } \\
\text { specialty, location of } \\
\text { patients PCP, potential } \\
\text { patient mileage needed to } \\
\text { travel for F2F consult with } \\
\text { specialist instead of } \\
\text { eConsult and specialty } \\
\text { care use following } \\
\text { eConsult }\end{array}$ & Moderate \\
\hline Liddy $^{21}$ & 2015 & Canada & Multispecialty & Mixed-methods & 2,052 eConsults & $\begin{array}{l}\text { Satisfaction, education, } \\
\text { impact on patient care, } \\
\text { response time }\end{array}$ & Weak \\
\hline Liddy $^{22}$ & 2015 & Canada & Multispecialty & $\begin{array}{l}\text { Economic analysis } \\
\text { based on survey }\end{array}$ & $\begin{array}{l}235 \text { PCPs, } 27 \\
\text { specialists }\end{array}$ & Cost savings & Weak \\
\hline Liddy $^{23}$ & 2016 & Canada & Multispecialty & Costing evaluation & 3,487 eConsults & $\begin{array}{l}\text { Costs and potential } \\
\text { savings of eConsult based } \\
\text { on referral avoidance }\end{array}$ & Weak \\
\hline Liddy $^{31}$ & 2016 & Canada & Multispecialty & $\begin{array}{l}\text { Mixed methods } \\
\text { study (cross- } \\
\text { sectional analysis } \\
\text { and survey) }\end{array}$ & 4,260 eConsults & $\begin{array}{l}\text { eConsult patterns and } \\
\text { provider satisfaction }\end{array}$ & Weak \\
\hline Liddy $^{24}$ & 2016 & Canada & Multispecialty & Cross-sectional & 1,796 eConsults & $\begin{array}{l}\text { Response time, impact of } \\
\text { eConsult on course of } \\
\text { action, Provider/patient } \\
\text { satisfaction }\end{array}$ & Weak \\
\hline Liddy $^{29}$ & 2016 & Canada & Multispecialty & Cross-sectional & 93 PCPs & $\begin{array}{l}\text { Response time, provider } \\
\text { satisfaction }\end{array}$ & Weak \\
\hline $\operatorname{Lin}^{46}$ & 2016 & Global (NMCP) & Multispecialty & $\begin{array}{l}\text { Teleconsultation } \\
\text { review }\end{array}$ & 585 Teleconsultations & $\begin{array}{l}\text { Referral/medevac } \\
\text { avoidance, response time, } \\
\text { cost savings }\end{array}$ & Weak \\
\hline Maddry ${ }^{38}$ & 2014 & $\begin{array}{l}\text { United States (note: } \\
\text { data obtained from } \\
\text { Iraq, Kuwait, and } \\
\text { Afghanistan also) }\end{array}$ & Multispecialty & $\begin{array}{l}\text { Retrospective } \\
\text { observational } \\
\text { analysis }\end{array}$ & 99 Teleconsultations & $\begin{array}{l}\text { Response time, type, and } \\
\text { frequency of } \\
\text { teleconsultation }\end{array}$ & Weak \\
\hline Marcolino ${ }^{33}$ & 2016 & Brazil & Multispecialty & Ecological study & $\begin{array}{l}\text { 73,698 } \\
\text { Teleconsultations }\end{array}$ & $\begin{array}{l}\text { Provider satisfaction, } \\
\text { avoidance of referrals, cost } \\
\text { savings }\end{array}$ & Weak \\
\hline Marcolino ${ }^{36}$ & 2015 & Brazil & Multispecialty & $\begin{array}{l}\text { Observational } \\
\text { retrospective study }\end{array}$ & $\begin{array}{l}63,975 \\
\text { Teleconsultations }\end{array}$ & $\begin{array}{l}\text { Referral avoidance, } \\
\text { response time, provider } \\
\text { satisfaction }\end{array}$ & Weak \\
\hline McGeady ${ }^{43}$ & 2014 & United States & Multispecialty & $\begin{array}{l}\text { Retrospective chart } \\
\text { review }\end{array}$ & $\begin{array}{l}\text { 1,705 eReferrals, } 487 \\
\text { patients }\end{array}$ & $\begin{array}{l}\text { Avoidance of referrals, } \\
\text { diagnostic testing } \\
\text { efficiency }\end{array}$ & Weak \\
\hline
\end{tabular}




\begin{tabular}{|c|c|c|c|c|c|c|c|}
\hline $\begin{array}{c}\text { FIRST } \\
\text { AUTHOR }\end{array}$ & YEAR & SETTING & $\begin{array}{l}\text { TYPE OF } \\
\text { SERVICE }\end{array}$ & $\begin{array}{l}\text { STUDY } \\
\text { DESIGN }\end{array}$ & $\begin{array}{c}\text { STUDY } \\
\text { POPULATION }\end{array}$ & OUTCOMES & $\begin{array}{l}\text { QUALITY } \\
\text { RATING }\end{array}$ \\
\hline Murthy ${ }^{27}$ & 2017 & Canada & Multispecialty & $\begin{array}{l}\text { Retrospective } \\
\text { analysis utilizing } \\
\text { mixed methods }\end{array}$ & $\begin{array}{l}224 \text { Infectious } \\
\text { diseases eConsults }\end{array}$ & $\begin{array}{l}\text { eConsult response time, } \\
\text { impact on course of } \\
\text { action, provider } \\
\text { satisfaction, impact on } \\
\text { patient care }\end{array}$ & Weak \\
\hline $\mathrm{Nami}^{50}$ & 2015 & Italy and Austria & Single specialty & Clinical trial & 391 Patients & $\begin{array}{l}\text { Concordance between } \\
\text { store and forward and } \\
\text { face-to-face consultation } \\
\text { management }\end{array}$ & Weak \\
\hline Nelson ${ }^{49}$ & 2016 & United States & Single specialty & Prospective study & $\begin{array}{l}196 \text { Teledermatology } \\
\text { consults }\end{array}$ & $\begin{array}{l}\text { Impact on diagnosis and } \\
\text { management (response } \\
\text { time, anticipated level of } \\
\text { dermatology input } \\
\text { without teledermatology, } \\
\text { and number of consults } \\
\text { managed solely with } \\
\text { teledermatology) }\end{array}$ & Weak \\
\hline North $^{10}$ & 2015 & United States & Multispecialty & Retrospective review & $\begin{array}{l}3,008 \text { eConsults, } 2,885 \\
\text { patients, } 353 \\
\text { specialists }\end{array}$ & $\begin{array}{l}\text { Conversion of eConsults to } \\
\text { face-to-face assessments }\end{array}$ & Weak \\
\hline Olayiwola ${ }^{39}$ & 2016 & United States & Single specialty & $\begin{array}{l}\text { Cluster-randomized } \\
\text { controlled trial }\end{array}$ & $\begin{array}{l}19 \text { PCPs (control), } 17 \\
\text { PCPs (intervention) }\end{array}$ & $\begin{array}{l}\text { Specialist response time, } \\
\text { patient outcomes (ED } \\
\text { utilization), physician } \\
\text { satisfaction, workload }\end{array}$ & Moderate \\
\hline Pecina $^{18}$ & 2016 & United States & Multispecialty & Retrospective study & 1,041 eConsults & $\begin{array}{l}\text { Follow-up of } \\
\text { recommendation from the } \\
\text { e-consultations by the } \\
\text { PCP }\end{array}$ & Weak \\
\hline Pecina $^{19}$ & 2016 & United States & Multispecialty & $\begin{array}{l}\text { Retrospective } \\
\text { analysis }\end{array}$ & 5,115 eConsults & $\begin{array}{l}\text { Reason for face-to-face } \\
\text { referrals following } \\
\text { eConsult }\end{array}$ & Weak \\
\hline Price $^{40}$ & 2016 & United States & Multispecialty & Chart review & 266 eReferrals & $\begin{array}{l}\text { Reasons for not } \\
\text { scheduling referrals, harm, } \\
\text { and potential for harm } \\
\text { caused by preconsultation }\end{array}$ & Weak \\
\hline Rodriguez $^{13}$ & 2015 & United States & Multispecialty & $\begin{array}{l}\text { Quality improvement } \\
\text { project evaluation: } \\
\text { semistructured } \\
\text { telephone interviews }\end{array}$ & $\begin{array}{l}15 \text { Veteran patients, } \\
15 \text { PCPs, } 4 \text { specialists }\end{array}$ & $\begin{array}{l}\text { Provider satisfaction, } \\
\text { patient satisfaction }\end{array}$ & Strong \\
\hline Scheibe ${ }^{51}$ & 2015 & United States & Multispecialty & $\begin{array}{l}\text { Retrospective chart } \\
\text { review }\end{array}$ & 2,105 eReferrals & $\begin{array}{l}\text { Volume/appropriateness } \\
\text { of pre-eConsultation } \\
\text { exchange and impact on } \\
\text { face-to-face referral }\end{array}$ & Moderate \\
\hline Segura ${ }^{47}$ & 2016 & Spain & Multispecialty & $\begin{array}{l}\text { Observational } \\
\text { retrospective study }\end{array}$ & $\begin{array}{l}938 \text { Virtual consults, } \\
44 \text { PCPs }\end{array}$ & $\begin{array}{l}\text { Avoidance of referrals, } \\
\text { provider satisfaction }\end{array}$ & Weak \\
\hline Shehata ${ }^{28}$ & 2016 & Canada & Multispecialty & $\begin{array}{l}\text { Retrospective } \\
\text { electronic chart } \\
\text { review }\end{array}$ & $\begin{array}{l}394 \text { eConsults } 151 \\
\text { PCP }\end{array}$ & $\begin{array}{l}\text { Avoidance of traditional } \\
\text { referrals }\end{array}$ & Weak \\
\hline
\end{tabular}




\section{SYSTEMATIC REVIEW OF ECONSULT SERVICES}

\begin{tabular}{|c|c|c|c|c|c|c|c|}
\hline $\begin{array}{c}\text { FIRST } \\
\text { AUTHOR }\end{array}$ & YEAR & SETTING & $\begin{array}{l}\text { TYPE OF } \\
\text { SERVICE }\end{array}$ & $\begin{array}{l}\text { STUDY } \\
\text { DESIGN }\end{array}$ & $\begin{array}{c}\text { STUDY } \\
\text { POPULATION }\end{array}$ & OUTCOMES & $\begin{array}{l}\text { QUALITY } \\
\text { RATING }\end{array}$ \\
\hline Shipherd ${ }^{11}$ & 2016 & United States & Multispecialty & $\begin{array}{l}\text { Feasibility program } \\
\text { evaluation }\end{array}$ & $\begin{array}{l}303 \text { eConsults, } 230 \\
\text { patients }\end{array}$ & $\begin{array}{l}\text { Time spent responding to } \\
\text { econsults, question types }\end{array}$ & Weak \\
\hline $\operatorname{Tran}^{26}$ & 2016 & Canada & Multispecialty & Cross-sectional & 1,055 eConsults & $\begin{array}{l}\text { Impact of question type } \\
\text { on avoidance of referrals } \\
\text { and discrepancies between } \\
\text { PCPs and specialists }\end{array}$ & Weak \\
\hline $\operatorname{Tran}^{32}$ & 2016 & Canada & Multispecialty & Cross-sectional & $\begin{array}{l}180 \text { PCPs, } 464 \\
\text { eConsults }\end{array}$ & $\begin{array}{l}\text { Provider satisfaction, } \\
\text { response time, frequency } \\
\text { of additional } \\
\text { recommendations by } \\
\text { specialist }\end{array}$ & Moderate \\
\hline Thijssing $^{37}$ & 2014 & The Netherlands & Single specialty & Descriptive study & $\begin{array}{l}227 \text { Telespirometry } \\
\text { tests, 4,488 } \\
\text { telepulmonology } \\
\text { consultations }\end{array}$ & $\begin{array}{l}\text { Referral avoidance, impact } \\
\text { on patient care, and } \\
\text { education for provider }\end{array}$ & Weak \\
\hline Wrenn $^{16}$ & 2017 & United States & Multispecialty & $\begin{array}{l}\text { Retrospective } \\
\text { descriptive analysis }\end{array}$ & $\begin{array}{l}200 \text { eConsults, } 86 \\
\text { PCPs, } 195 \text { patients }\end{array}$ & $\begin{array}{l}\text { Referrals following } \\
\text { eConsult, frequency of } \\
\text { question type/answers, } \\
\text { frequency of PCP } \\
\text { following } \\
\text { recommendation }\end{array}$ & Weak \\
\hline
\end{tabular}

MSF, Médecins Sans Frontières; NMCP, Naval Medical Center Portsmouth; PCPs, primary care providers; VHA, Veterans Health Administration.

authors noted that scheduling of appointments may not have avoided this harm. Within unresolved referrals (no evidence that the referral complaints resolved spontaneously or were addressed in some other way), 55 (73\%) were identified as having potential for major harm (including prolonged hospitalizations, permanent disability, delayed diagnosis of malignancy, and/or death). ${ }^{40}$ The authors concluded that the few adverse outcomes that were observed were not due to communication lapses in the referral process, but rather to being unintentionally left unscheduled with evidence for discontinuity of care and lack of patient or provider follow-up. A retrospective analysis of dermatology cases referred by MSF field doctors revealed that only 10 of 65 cases (15\%) had patient follow-up data with two cases of patient death. ${ }^{41}$ The lack of information about patient follow-up was a critical issue raised by the participating specialists and referrers. ${ }^{41}$ Similar lack of follow-up was noted by Byrom et al., who reported that patient outcome was largely unknown in 83\% of 406 cases. $^{42}$

\section{PATIENT EXPERIENCE OF CARE}

Thirty (out of 43) studies reported on percentage of avoided face-to-face visits. The lowest was $7.4 \%{ }^{43}$ and the highest
$78 \%,{ }^{36}$ with most studies reporting between $22 \%$ and $68 \%$ reductions in face-to-face specialist visits. Barnet et al. noted a threefold variation in the rate of eConsult requests resolved without a visit across specialist consultants. ${ }^{44}$

Only one study described interviewing patients (in addition to providers), who had an eConsult, about their experience, including satisfaction with the eConsult program and perceived facilitators of and barriers to eConsult utilization. Overall, the patients were satisfied with the eConsult program (median ratings of 5 on a 5-point Likert scale) in terms of met expectations and confidence in eConsult. ${ }^{13}$ They also rated the service high (median ratings of 4) for quality of care, timeliness, improved access, and safety. Furthermore, patients identified communication (effective communication with PCPs or providers) as the domain that was most important regarding their overall satisfaction with the eConsult process and unanimously indicated that they intended to use eConsults in the future based on the quality of care and the timeliness of care. ${ }^{13}$ Other studies surveyed users (PCP referrers and specialists) to examine the services' benefits for patients. Bonnardot et al. showed that 79\% of referrers reported that the advice received improved their management of the patient. ${ }^{45}$ Similarly, Thijssing et al. reported that in $72 \%$ of the cases, the 


\section{LIDDY ET AL.}

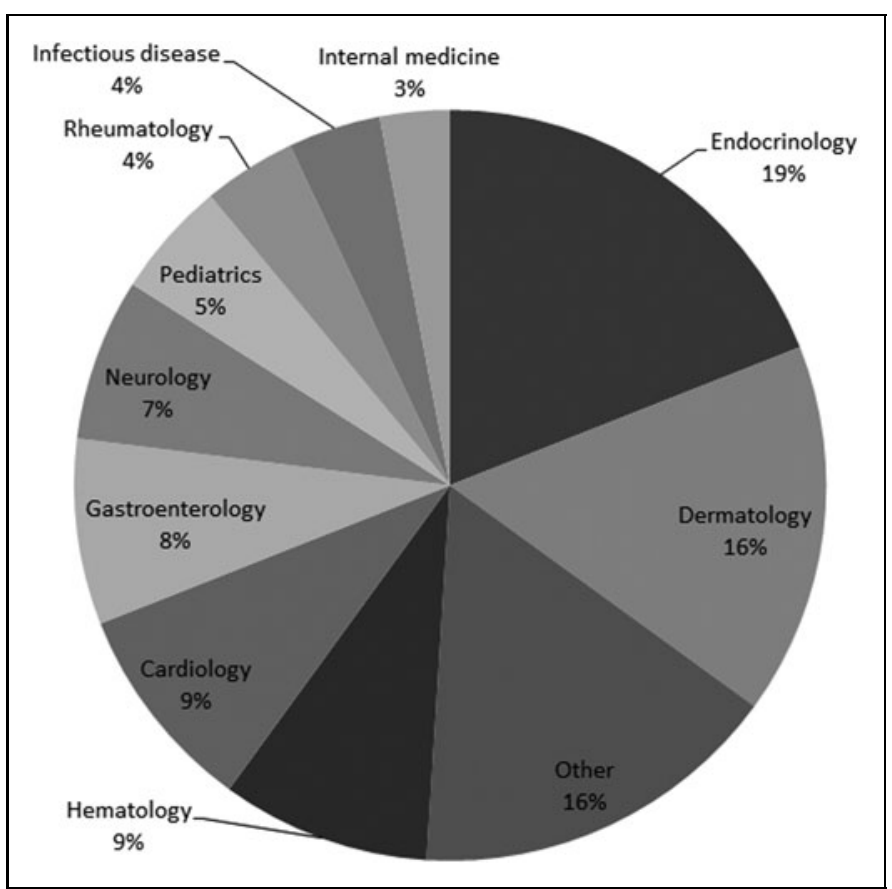

Fig. 2. Proportions of specialties accessed via eConsult service (based on the top three specialties assessed most frequently in each article). Note: "other" refers to small proportions ( $1 \%$ each) for the following specialties: Chronic Back Pain, Gastroenterology and Hepatology, Medical Toxicology, Obstetrics and Gynecology, Orthopedics, Psychiatry, Pulmonology and Spirometry, Radiology, Sleep medicine, Spine Center, Transgender Care General, and Urology.

referrers indicated that they and the patients were helped by the consultant's response. ${ }^{37}$ In three separate studies from the Champlain BASE service, primary healthcare providers (i.e., nurse practitioners and/or physicians) were asked to rate the value of eConsult to their patients using 5-point Likert scale (from 1: little/no value to 5: very high value). ${ }^{24,29,31}$ The findings were consistently positive, with most providers (89\%) rating eConsult's value to patients as high or very high (i.e., 4 or 5 out of 5).

\section{REDUCING PER CAPITA COST OF CARE}

Six articles, including two by our study group, reported on costs of eConsults (Table 5). Different techniques were used that included cost analysis, cost-minimization analysis (comparing the costs of eConsult with traditional referral methods), and cost effectiveness (determination of a savings ratio, such as a return on investment or ROI). Studies comparing costs (i.e., those conducting a cost-minimization analysis) of eConsults with in-person specialist visits report eConsult costs (viewed through impact on health services) ranging from $\$ 5$ per eConsult (compared with $\$ 56$ for face-to- face) ${ }^{33}$; to $\$ 298$ per person using eConsult (compared with $\$ 338$ for face-to-face). ${ }^{14}$ Alternatively, another study estimated monthly savings for the military (using a cost minimization analysis) by using eConsult to be $\$ 140,907$ (yearly savings were $\$ 467,181$ ); where civilian monthly savings were estimated as $\$ 28,260$ (yearly savings were $\$ 105,400$ ). ${ }^{46}$ The return of investment was also estimated at 6.1 for eConsult in one study assessing cost effectiveness. ${ }^{33}$ From a societal perspective, another study found (through a cost minimization analysis) that costs of eConsult averaged at $\$ 460$ per patient, compared with face-to-face costs of $\$ 542$ per patient. ${ }^{14}$ Similarly, in another study, societal savings (including cost to the healthcare payer, and costs to the patient) were estimated to be approximately $\$ 11$ per eConsult. ${ }^{23}$ Finally, another study suggested a potential for millions in transportation saving by implementing eConsult to minimize face-toface consultations. ${ }^{12}$

\section{PROVIDER EXPERIENCE OF CARE}

Many studies assessed provider experiences of eConsult, including perceived value, satisfaction, educational value, quality of response, and impact on workload. There are several reports from Champlain BASE assessing perceived value using a 5-point Likert scale. Overall, more than 90\% of providers rated eConsult as having a high to very high value for themselves (i.e., at a value of at least 4 out of 5). ${ }^{24,29,31}$ In other studies, when asked about satisfaction with eConsult, 75$100 \%$ of providers reported being satisfied, ${ }^{33,47}$ and most providers reported that they would use eConsult again in the future. $^{13}$

eConsult's educational value was noted in several studies, with $90 \%$ of providers reported having learned from eConsult, ${ }^{37}$ whereas in others $74 \%$ reported their questions were answered, ${ }^{35}$ and $89 \%$ thought eConsult results were conclusive ${ }^{47}$ Providers also reported that psychiatric eConsults increased access to specialty consultations for mental health and improved support for diagnosis and treatment. ${ }^{48}$ In another study, 75\% of providers reported no impact or decreased workload as a result of eConsult. ${ }^{39}$ In general, the time it took for the specialists to prepare an answer to the question from an eConsult ranged from under $20 \mathrm{~min},{ }^{24}$ to under $30 \mathrm{~min},{ }^{15}$ to 78 min. $^{11}$

Many studies also evaluated timeliness of eConsult. The average/median time for the PCPs to receive a response from the specialists to the eConsult requests ranged from $<1^{18,49,50}$ to $<6$ days. ${ }^{11,51}$ In a cardiology RCT, which compared time to response between eConsult and traditional referrals, the median number of days to receive a response was 5 days for eConsults versus 24 days for traditional referrals. ${ }^{39}$ Only one 


\section{SYSTEMATIC REVIEW OF ECONSULT SERVICES}

Table 3. Service/Article Breakdown

\section{SERVICE}

ARTICLES

Champlain BASE ${ }^{\mathrm{TM}}$ eConsult Service

\begin{tabular}{|c|c|}
\hline & \\
\hline & Johnston et al. $(2017)^{25}$ \\
\hline & Keely et al. $(2015)^{30}$ \\
\hline & Liddy et al. $(2015)^{21}$ \\
\hline & Liddy et al. $(2015)^{22}$ \\
\hline & Liddy et al. (2016) ${ }^{29}$ \\
\hline & Liddy et al. $(2016)^{23}$ \\
\hline & Liddy et al. $(2016)^{24}$ \\
\hline & Liddy et al. $(2016)^{31}$ \\
\hline & Murthy et al. $(2017)^{27}$ \\
\hline & Shehata et al. $(2016)^{28}$ \\
\hline & Tran et al. $(2016)^{32}$ \\
\hline & Tran et al. $(2016)^{26}$ \\
\hline TNMG & Alkmim et al. $(2015)^{34}$ \\
\hline & Alkmim et al. $(2015)^{35}$ \\
\hline & Marcolino et al. $(2016)^{33}$ \\
\hline & Marcolino et al. $(2015)^{36}$ \\
\hline Mayo Clinic eConsult & North et al. $(2015)^{10}$ \\
\hline & Pecina and North (2016) ${ }^{19}$ \\
\hline & Pecina et al. $(2016)^{18}$ \\
\hline SFGH eConsult & McGeady et al. (2014) $)^{43}$ \\
\hline & Price et al. $(2016)^{40}$ \\
\hline & Scheibe et al. $(2015)^{51}$ \\
\hline UCSF eConsult & Cruz et al. $(2015)^{17}$ \\
\hline & Wrenn et al. $(2016)^{16}$ \\
\hline MSF Telemedicine Network & Bonnardot et al. (2014) ${ }^{45}$ \\
\hline & Delaigue et al. $(2014)^{41}$ \\
\hline VHA (nationwide) & Kirsh et al. $(2015)^{12}$ \\
\hline & Shipherd et al. (2016) $)^{11}$ \\
\hline $\begin{array}{l}\text { Los Angeles Safety Net Program DHS (Los } \\
\text { Angeles County Department of Health } \\
\text { Services) eConsult }\end{array}$ & Barnett et al. $(2017)^{44}$ \\
\hline $\begin{array}{l}\text { KP Safety Net Specialty Care Program } \\
\text { (Denver) eConsult (collaboration with KPCO) }\end{array}$ & Fort et al. $(2017)^{52}$ \\
\hline Allina Health Twin Cities eConsult & Golberstein et al. $(2017)^{48}$ \\
\hline $\begin{array}{l}\text { U.S. Army Medical Department (AMEDD) AKO } \\
\text { electronic mail system for dermatology; } \\
\text { Electronic mail telemedicine system }\end{array}$ & Maddry et al. $(2014)^{38}$ \\
\hline
\end{tabular}

continued $\rightarrow$

\begin{tabular}{|c|c|}
\hline SERVICE & ARTICLES \\
\hline $\begin{array}{l}\text { HELP Teleconsultations; Pediatric and Adult } \\
\text { (military and civilian families) }\end{array}$ & Lin et al. $(2016)^{46}$ \\
\hline $\begin{array}{l}\text { Department Dermatology Siena University/ } \\
\text { Medical University STF/Store-and-Forward } \\
\text { Mobile Teledermatology using MugDerma }\end{array}$ & Nami et al. $(2015)^{50}$ \\
\hline $\begin{array}{l}\text { University of Pennsylvania Store-and- } \\
\text { Forward Teledermatology: AccessDerm mobile } \\
\text { SAF platform }\end{array}$ & Nelson et al. $(2016)^{49}$ \\
\hline $\begin{array}{l}\text { Tele-Derm National: Australian College of } \\
\text { Rural and Remote Medicine (ACRRM) }\end{array}$ & Byrom et al. $(2016)^{42}$ \\
\hline $\begin{array}{l}\text { Netherlands Telepulmonology and } \\
\text { Telespirometry KSYOS Telemedical Center }\end{array}$ & Thijssing et al. $(2014)^{37}$ \\
\hline $\mathrm{CHCl}$ eConsult & Olayiwola et al. $(2016)^{39}$ \\
\hline $\begin{array}{l}\text { Autonomous Community of the Canary } \\
\text { Islands Virtual Consultations in } \\
\text { Rheumatology (DRAGO) }\end{array}$ & Segura and Bustabad $(2016)^{47}$ \\
\hline $\begin{array}{l}\text { Veterans Affairs Boston Healthcare System } \\
\text { (VABHS) }\end{array}$ & Gupte et al. $(2016)^{15}$ \\
\hline VA Minneapolis & Datta et al. $(2015)^{14}$ \\
\hline $\begin{array}{l}\text { Veterans Affairs Pittsburg Health Care System } \\
\text { (VAPHS) }\end{array}$ & Rodriguez et al. $(2015)^{13}$ \\
\hline
\end{tabular}

AKO, Army Knowledge Online; $\mathrm{CHCl}$, Community Health Center, Inc.; DHS, Department of Health Services; HELP, Health Experts onLine at Portsmouth; KP, Kaiser Permanente; KPCO, Kaiser Permanente Colorado; SFGH, San Francisco General Hospital; TNMG, Telehealth Network of Minas Gerais; UCSF, University of California San Francisco; VA, Veterans Affairs.

study determined the median time for patient notification after eConsult and found it to be 3 days. ${ }^{18}$

Open-ended input responses revealed that most providers appreciated the service, finding it safe, timely, easy to use, beneficial to patient care, and capable of improving communication and facilitating provider education. ${ }^{25}$ Some challenges associated with eConsult were those relating to unclear directions from specialists, an occasional lack of information or pertinent questions delivered to specialist by the PCP, and lack of patient follow-up. ${ }^{41}$ Moreover, although the few studies which assessed specialist experiences found them to be positive (i.e., in terms of perceived impact on patient, ease of use, improving access, usefulness), one identified challenge was the potential for an increase in workload for specialists, along with the potential for issues in communication. ${ }^{30}$ Issues in communication refer to unclear specialist responses or expectations, which may be the result of unclear questions and expectations posed, ${ }^{15}$ or from an unclear 


\section{LIDDY ET AL.}

\section{Table 4. Description of Technological Platforms}

\section{PLATFORM TYPE}

LOCATION

\section{DESCRIPTION}

Shared health records

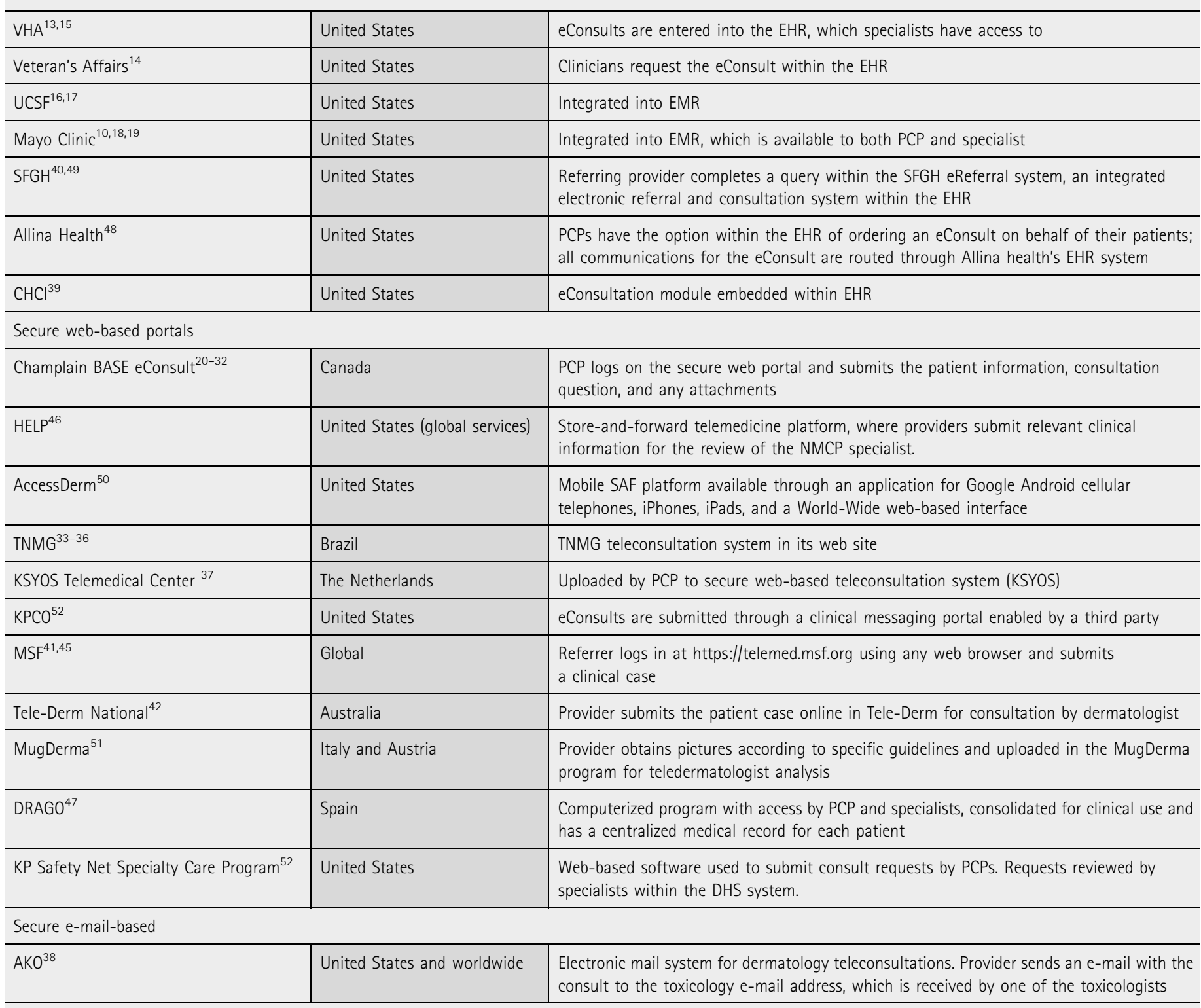

$E H R$, electronic health record; EMR, electronic medical record.

understanding on how to use the program. ${ }^{41}$ Fort et al. reported that many of the specialists participating in the Safety Net Specialty Care eConsult Program in Denver Metropolitan Area believed there was room to expand the program beyond the current volume of eConsults, although this perception varied by department, with high-volume or lower-staff-ratio departments expressing interest in maintaining the program at its current size. ${ }^{52}$

\section{Discussion}

In our updated systematic review of eConsult services worldwide, we viewed eConsult through the lens of the Quadruple Aim Framework, and attempted to assess the impact of this innovative technology on population health, patient experience of care (including quality and satisfaction), provider experience of providing care, and healthcare costs. Similarly to our past review, we found that, while the majority 


\begin{tabular}{|c|c|c|c|c|c|}
\hline Liddy 22 & 2015 & $\begin{array}{l}\text { Champlain BASE } \\
\text { eConsult Service }\end{array}$ & $\begin{array}{l}\text { (a) Traditional } \\
\text { (b) CA and CMA } \\
\text { (c) Health Service }\end{array}$ & $\begin{array}{l}\text { Assessing the direct (i.e., start-up/ } \\
\text { development costs) and variable costs } \\
\text { (associated with supporting eConsult } \\
\text { service; includes delivery and } \\
\text { consultation costs) of eConsultation. } \\
\text { Measured across } 3 \text { years. }\end{array}$ & $\begin{array}{l}\text { Total costs per eConsult for year } 1 \text { : } \\
\$ 131.05 \text {; year } 2 \text { : } \$ 10.34 \text {; year } 3: \$ 6.45 \text {. } \\
\text { Average cost across } 3 \text { years per } \\
\text { eConsult: } \$ 16.71 \text {. Compared with } \\
\text { average of } \$ 150 \text { per face-to-face visit. } \\
\text { eConsultation will break even when } \\
7,818 \text { eConsults are reached. }\end{array}$ \\
\hline $\operatorname{Lin}^{46}$ & 2016 & $\begin{array}{l}\text { HELP } \\
\text { teleconsultations; } \\
\text { Pediatric and Adult } \\
\text { (military and civilian } \\
\text { families) }\end{array}$ & $\begin{array}{l}\text { (a) Traditional } \\
\text { (b) CA and CMA } \\
\text { (c) Health Service }\end{array}$ & $\begin{array}{l}\text { Estimating cost savings by examining } \\
\text { the number of avoided medevacs (for } \\
\text { military) and specialist referrals (for } \\
\text { civilians). }\end{array}$ & $\begin{array}{l}\text { From June } 2014 \text { to May 2015, estimated } \\
\text { cost savings for military (assessed from } \\
\text { avoided medevacs) was } \$ 467,181 ; \text { cost } \\
\text { savings for civilians (assessed from } \\
\text { avoided specialist referrals) was } \\
\$ 105,400 \text {. Estimated monthly savings of } \\
\$ 140,907 \text { (from medevac prevention) } \\
\text { and } \$ 28,260 \text { (civilian referral } \\
\text { prevention). }\end{array}$ \\
\hline Liddy $^{23}$ & 2016 & $\begin{array}{l}\text { Champlain BASE } \\
\text { eConsult Service }\end{array}$ & $\begin{array}{l}\text { (a) Traditional } \\
\text { (b) CA and CMA } \\
\text { (c) Societal }\end{array}$ & $\begin{array}{l}\text { Potential societal costs and savings } \\
\text { assessed. Potential Societal costs: } \\
\text { include direct (costs to payer i.e., } \\
\text { delivery, consultation specific, and } \\
\text { referral costs) and indirect (costs to } \\
\text { patient). Potential Savings: include } \\
\text { direct (i.e., costs of avoided referrals) } \\
\text { and indirect costs (i.e., avoided travel } \\
\text { and productivity losses). }\end{array}$ & $\begin{array}{l}\text { From April } 2014 \text { to March } 2015 \text {, } \\
\text { eConsult societal costs (from } 3,487 \\
\text { eConsultations) estimated as } \$ 207,787 \text {; } \\
\text { estimated potential savings of } \\
\$ 246,516 \text {. Societal savings of } \$ 38,729 \\
\text { or } \$ 11 \text { per eConsult. }\end{array}$ \\
\hline Kirsh $^{12}$ & 2015 & $\begin{array}{l}\text { eConsultation } \\
\text { for the VHA }\end{array}$ & $\begin{array}{l}\text { (a) Traditional } \\
\text { (b) CA } \\
\text { (c) Health Service (VHA) }\end{array}$ & $\begin{array}{l}\text { Cost of travel for F2F consultation (VHA } \\
\text { reimburses } 41.5 \text { cents per mile) } \\
\text { analyzed for those receiving primary } \\
\text { care from community-based outpatient } \\
\text { clinics }(n=95,425) \text {. }\end{array}$ & $\begin{array}{l}\text { Patients would have to drive on average } \\
72.1 \text { miles for } F 2 F \text {, resulting in an } \\
\text { average cost of } \$ 2,853,387 \text {, suggesting } \\
\text { savings in this regard for using } \\
\text { eConsultation. }\end{array}$ \\
\hline Marcolino ${ }^{33}$ & 2016 & $\begin{array}{l}\text { Teleconsultation; } \\
\text { TNMG }\end{array}$ & $\begin{array}{l}\text { (a) Traditional } \\
\text { (b) CA, CMA, and CE } \\
\text { (c) Health Service }\end{array}$ & $\begin{array}{l}\text { Assessed number of referrals and } 2 \\
\text { main costs: Fixed (i.e., salaries and } \\
\text { benefits; not affected by referrals) and } \\
\text { variable costs (ex. Transportation) for } \\
2,538,697 \text { telehealth activities. From } \\
\text { this they calculated ROI. }\end{array}$ & $\begin{array}{l}\text { Cost for telehealth was } \$ 5.00 \text { USD, cost } \\
\text { of F } 2 F \text { was } \$ 56.00 \text {. Since } 80 \% \text { of F } 2 F \\
\text { referrals avoided, estimated savings } \\
\text { were } \$ 63 \text { million. Since } 10.3 \text { million } \\
\text { invested, ROI is } 6.1 \text { until Dec } 2015 \text {. }\end{array}$ \\
\hline
\end{tabular}

$C A$, cost analysis; $C E$, cost-effectiveness; $C M A$, cost-minimization analysis; $R O I$, return on investment. 


\section{LIDDY ET AL.}

of new research examined outcomes related to patient and provider experience, reporting on population health and cost measures is still limited. In contrast to the previous review, we have found an increased international presence of eConsult and noted that the breadth of specialty services offered has greatly expanded beyond dermatology, with most studies $(84 \%)$ focused on multispecialty and only a handful of studies addressing a single specialty service.

eConsult services in this review were shown to be effective in terms of providing faster access to specialists advice, with short response times of the participating specialists and resulting in substantial avoidance of face-to-face referral visits. The average/median time for specialists to respond to eConsult requests sent by PCPs ranged from 1 to 6 days. ${ }^{11,18,51}$ Comparing these wait times to those typically experienced with a traditional referral pathway for face-to-face visits with a specialist, which are at least 4 weeks in Canada, ${ }^{1}$ eConsults provide a promising alternative.

While a wider breadth of specialty services are now being offered by many eConsult services worldwide, which may enable a wider population to obtain access to specialty care, there are still significant gaps in understanding the impact of the service on population health using traditional measures of mortality, morbidity, and clinical outcomes. Only three articles in this systematic review provided some outcomes in relation to risk and mortality. While an RCT demonstrated the potential of eConsult for reducing adverse cardiovascular outcomes, ${ }^{39} 2$ retrospective studies reported potential for harm due to lack of patient follow-up..$^{40,41}$ As the number of patients receiving eConsults increases, linking to healthcare databases to determine impact may be beneficial, despite their shortcomings related to data availability and limitations in their ability to capture the details of clinical encounters across healthcare sectors. ${ }^{53,54}$

A majority of studies assessed patient experience of care from a PCP perspective, which points to a need to measure patient experience directly. In our own recent study, we interviewed patients who have had an eConsult done on their behalf by their PCP and reported that all patients expressed acceptance for eConsult as a model for improving access to specialist care, had largely positive experiences with it as a model of care delivery, and supported its use in their future care. ${ }^{55}$ It is important that future research includes the patient voice through the incorporation of patient-reported outcomes and the direct measurement of the patient experiences with services such as eConsult that have direct impact on the quality of care they receive.

Findings in this review support the view that eConsult improves the work life of healthcare providers as per the Quadruple
Aim Framework. Unlike other e-health technology solutions, eConsult services show sustained use and spread, high adoption, and little pushback from the providers who use them, as evidenced by high provider satisfaction ratings. ${ }^{25}$ Furthermore, reports of provider education, quality of responses/feedback, and impact on healthcare support and workload, ${ }^{37}$ suggest the provider experience is generally positive in these domains and point out that eConsult has the potential to improve overall job satisfaction, retention in remote communities, and provide educational/continuing professional development opportunities.

Although the articles analyzed in this review shed a considerable amount of light on the prevalence and impacts of eConsult, there are a number of limitations to consider. First, the paucity of RCTs reflects the early, emerging nature of this health technology. There is a need for more studies with stronger designs, including more traditional measures of population health. Similarly, the impact of eConsult services on specialists, although deemed fairly positive by a few articles, is another understudied area in need of further attention, especially since there is a potential for increases in workload, which could affect quality of care. ${ }^{30}$ Finally, in agreement with our previous review, the question of cost effectiveness continues to be underexamined. Only six studies attempted to address and report on the economic impact of eConsult services. All used different methods, ranging from cost analysis, through cost-minimization analysis, to cost effectiveness. In general, these studies yield promising findings in terms of cost savings to the healthcare systems, return of investment, and societal savings. Regardless, studies like those assessed in this review, are useful in assessing and adding to our current understanding, and can be used as an exploratory base for further research.

\section{Conclusions}

We assessed the breadth of literature on eConsult through the lens of the Quadruple Aim Framework to guide our narrative synthesis and attempted to assess the impact of this innovative technology on population health, patient experience of care (including quality and satisfaction), provider experience of providing care, and healthcare costs. We characterized the various technology platforms used and confirmed that they still range from services built into existing shared electronic health systems to stand-alone web-based portals. The results to date indicate that eConsult systems have expanded significantly internationally and are largely multiservice in nature. While the majority of new research examined outcomes related to patient and provider experience, the population health and cost measures continue to be limited and require further development. 


\section{SYSTEMATIC REVIEW OF ECONSULT SERVICES}

\section{Acknowledgments}

The authors would like to thank Canada Health Infoway for providing financial support for the students who helped conduct this study. Additional funding for this study was provided by the Ontario Ministry of Health and Long-Term Care and the Canadian Institutes for Health Research. The funders were not involved in the study design, data collection, data analysis, or article preparation, or in the decision to publish the results. The views expressed do not necessarily reflect those of the Province of Ontario. For the purposes of transparency, the authors would also like to note that their service, the Champlain BASE eConsult service, was among the programs identified by this review.

\section{Disclosure Statement}

The authors have no conflicts of interest to declare, real or perceived.

\section{REFERENCES}

1. Canadian Institute for Health Information. How Canada compares: Results from the Commonwealth Fund's 2016 International Health Policy Survey of Adults in 11 countries. Ottawa: $\mathrm{CIHI}$, 2017. www.cihi.ca/sites/default/files/document/textalternative-version-2016-cmwf-en-web.pdf (last accessed March 21, 2018).

2. NHS England. Statistical Press Notice: NHS referral to treatment (RTT) waiting times data August 2017. London: NHS, 2017. www.england.nhs.uk/statistics/ wp-content/uploads/sites/2/2017/06/RT-statistical-press-notice-PDF-300K51515.pdf (last accessed March 21, 2018).

3. UK Department of Health. Northern Ireland waiting time statistics: Outpatient waiting times quarter ending June 2017. London: UK Department of Health, 2017. www.health-ni.gov.uk/sites/default/files/publications/health/hs-niwtsoutpatient-waiting-times-q1-17-18.pdf (last accessed March 21, 2018).

4. Juliani C, MacPhee M, Spiri W. Brazilian specialists perspectives on the patient referral process. Healthcare (Basel) 2017;5:4

5. Liddy $C$, Drosinis $P$, Keely E. Electronic consultation systems: Worldwide prevalence and their impact on patient care-A systematic review. Fam Pract 2016;33:274-285

6. Vimalananda VG, Gupte G, Seraj SM, Orlander J, Berlowitz D, Fincke BG, et al. Electronic consultations (e-consults) to improve access to specialty care: A systematic review and narrative synthesis. J Telemed Telecare 2015;21:323-330.

7. Bodenheimer $T$, Sinsky $C$. From triple to quadruple aim: Care of the patient requires care of the provider. Ann Fam Medicine 2014;12:573-576.

8. Thomas BH, Ciliska D, Dobbins M, Micucci S. A process for systematically reviewing the literature: Providing the research evidence for public health nursing interventions. Worldviews Evid Based Nurs 2004;1:176-184.

9. Berwick D, Nolan T, Whittington. The triple aim: Care, health and cost. Health Aff 2008;27:759-769.

10. North F, Uthke LD, Tulledge-Scheitel SM. Internal e-consultations in an integrated multispecialty practice: A retrospective review of use, content, and outcomes. J Telemed Telecare 2015;21:151-159.

11. Shipherd JC, Kauth MR, Matza A. Nationwide interdisciplinary e-consultation on transgender care in the Veterans Health Administration. J Telemed E Health 2016;22:1008-1012.

12. Kirsh S, Carey E, Aron DC, Cardenas O, Graham G, Jain R, et al. Impact of a national specialty e-consultation implementation project on access. Am J Manag Care 2015;21:e648-e654.
13. Rodriguez KL, Burkitt KH, Bayliss NK, Skoko JE, Switzer GE, Zickmund SL, et al. Veteran, primary care provider, and specialist satisfaction with electronic consultation. JMIR Med Inform 2015;3:e5.

14. Datta SK, Warshaw EM, Edison KE, Kapur K, Thottapurathu L, Moritz TE, et al. Cost and utility analysis of a store-and-forward teledermatology referral system: A randomized clinical trial. JAMA Derm 2015;151:1-7.

15. Gupte G, Vimalananda V, Simon SR, DeVito K, Clark J, Orlander JD. Disruptive innovation: Implementation of electronic consultations in a Veterans Affairs Health Care System. JMIR Med Inform 2016;4:e6.

16. Wrenn $\mathrm{K}$, Catschegn $\mathrm{S}$, Cruz M, Gleason N, Gonzales R. Analysis of an electronic consultation program at an academic medical centre: Primary care provider questions, specialist responses, and primary care provider actions. $J$ Telemed Telecare 2017;23:217-224.

17. Cruz ML, Gleason N, Wang M, Wrenn K, Gonzales R. Transforming the endocrine consult: Asynchronous provider consultations. Endocr Pract 2015; 21:514-521.

18. Pecina JL, Frank JM, North F. A retrospective study on how primary care providers manage specialists' recommendations after an e-consultation. SAGE Open Med 2016;4:2050312116682127.

19. Pecina JL, North F. Early e-consultation face-to-face conversions. J Telemed Telecare 2016;22:269-276.

20. Fogel A, Khamisa K, Afkham A, Liddy C, Keely E. Ask the eConsultant: Improving access to haematology expertise using an asynchronous eConsult system. $J$ Telemed Telecare 2016;23:421-427.

21. Liddy C, Afkham A, Drosinis P, Joschko J, Keely E. Impact and satisfaction with a new eConsult service: A mixed methods study of primary care providers. $J$ Am Board Fam Med 2015;28:394-403.

22. Liddy C, Deri Armstrong C, Drosinis P, Mito-Yobo F, Afkham A, Keely E. What are the costs of improving access to specialists through eConsultation? The Champlain BASE experience. Stud Health Technol Inform 2015;209:75-83.

23. Liddy C, Drosinis P, Deri Armstrong C, McKellips F, Afkham A, Keely E. What are the cost savings associated with providing access to specialist care through the Champlain BASE eConsult service? A costing evaluation. BMJ Open 2016; 6:e010920.

24. Liddy C, Drosinis P, Joschko J, Keely E. Improving access to specialist care for an aging population. Gerontol Geriatr Med 2016;2:1-7.

25. Johnston DL, Murto K, Kurzawa J, Liddy C, Keely E, Lai L. Use of electronic consultation system to improve access to care in pediatric hematologyl oncology. J Pediatr Hematol Oncol 2017;39:e367-e369.

26. Tran C, Liddy C, Pinto N, Keely E. Impact of question content on e-consultation outcomes. Telemed J E Health 2016;22:216-222.

27. Murthy R, Rose G, Liddy C, Afkham A, Keely E. eConsultations to infectious disease specialists: Questions asked and impact on primary care providers' behavior. Open Forum Infect Dis 2017;4:0fx030.

28. Shehata F, Posner G, Afkham A, Liddy C, Keely E. Evaluation of an electronic consultation service in obstetrics and gynecology in Ontario. Obstetr Gynecol 2016;127:1033-1038.

29. Liddy C, Smyth C, Poulin PA, Joschko J, Rebelo M, Keely E. Improving access to chronic pain services through econsultation: A cross-sectional study of the Champlain BASE eConsult Service. Pain Med 2016;17:1049-1057.

30. Keely E, Drosinis P, Afkham A, Liddy C. Perspectives of Champlain BASE specialist physicians: Their motivation, experiences and recommendations for providing eConsultations to primary care providers. Stud Tech Health Inform 2015;209:38-45

31. Liddy C, Deri Armstrong C, McKellips F, Keely E. A comparison of referral patterns to a multispecialty eConsultation service between nurse practitioners and family physicians: The case for eConsult. J Am Assoc Nurse Pract 2016;28:144-150.

32. Tran CS, Liddy CE, Liu DM, Afkham A, Keely EJ. eConsults to endocrinologists improve access and change primary care provider behavior. Endocr Pract 2016;22:1145-1150. 


\section{LIDDY ET AL.}

33. Marcolino M, Minelli Figueira R, Pereira Afonso dos Santos J, Silva Cardoso C, Luiz Ribeiro A, Alkmim MB. The experience of a sustainable large scale Brazilian telehealth network. Telemed J E Health 2016;22:899-908.

34. Alkmim MB, Marcolino MS, Figueira RM, Sousa L, Nunes MS, Cardoso CS, et al. Factors associated with the use of a teleconsultation system in Brazilian primary care. J Telemed E Health 2015;21:473-483.

35. Alkmim MB, Marcolino MS, Maia JX, Pessoa CG, Machado E, Sousa L. Clinical quality control of a large-scale teleconsultation service. Stud Health Technol Inform 2015;216:988.

36. Marcolino MS, Alkmim MB. Teleconsultations to provide support for primary care practitioners and improve quality of care-The experience of a large scale telehealth service in Brazil. Stud Health Technol Inform 2015; 216:987.

37. Thijssing $L$, van der Heijden JP, Melissant CF, Chavannes NH, Witkamp $L$, Jaspers MW. Telepulmonology and telespirometry. In: Lovis C, et al., eds. e-Health-For continuity of care. Amsterdam: IOS Press; 2014:211-215.

38. Maddry JK, Sessions D, Heard K, Lappan C, McManus J, Bebarta VS. Wartime toxicology: Evaluation of a military medical toxicology telemedicine consults service to assist physicians serving overseas and in combat (2005-2012). J Med Toxicol 2014;10:261-265.

39. Olayiwola JN, Anderson D, Jepeal N, Aseltine R, Pickett C, Yan J, et al. Electronic consultations to improve the primary care-specialty care interface for cardiology in the medically underserved: A cluster-randomized controlled trial. Ann Fam Med 2016;14:133-140.

40. Price EL, Sewell JL, Chen AH, Sarkar U. Minding the gaps: Assessing communication outcomes of electronic preconsultation exchange. Jt Comm J Qual Patient Saf 2016;42:341-354.

41. Delaigue S, Morand JJ, Olson D, Wootton R, Bonnardot L. Teledermatology in low-resource settings: The MSF experience with a multilingual tele-expertise platform. Front Public Health 2014;14;2:233.

42. Byrom L, Lucas L, Sheedy V, Madison K, Mclver L, Castrisos G, et al. Tele-Derm National: A decade of teledermatology in rural and remote Australia. Aust J Rural Health 2016;24:193-199.

43. McGeady JB, Blaschko SD, Brajtbord JS, Sewell JL, Chen AH, Breyer BN. Electronic preconsultation as a method of quality improvement for urological referrals. Urolog Pract 2014;1:172-175.

44. Barnett ML, Yee HF, Mehrotra A, Giboney P. Los Angeles safety-net program eConsult system was rapidly adopted and decreased wait times to see specialists. Health Aff 2017;36:492-499.

45. Bonnardot L, Liu J, Wootton E, Amoros I, Olson D, Wong S, et al. The development of a multilingual tool for facilitating the primary-specialty care interface in low resource settings: The MSF tele-expertise system. Front Pub Health $2014 ; 2: 126$.

46. Lin $\mathrm{AH}$, Cole JH, Chin JC, Mahnke CB. The Health Experts onLine at Portsmouth (HELP) system: One-year review of adult and Pediatric Asynchronous Telehealth Consultations. SAGE Open Med 2016;4: 2050312115626433.
47. Segura BT, Bustabad S. A new form of communication between rheumatology and primary care: The virtual consultation. Reumatol Clin 2016;12:11-14.

48. Golberstein E, Kolvenbach S, Carruthers H, Druss B, Goering P. Effects of electronic psychiatric consultations on primary care provider perceptions of mental health care: Survey results from a randomized evaluation. Healthc (Amst) 2018;6:17-22.

49. Nelson CA, Takeshita J, Wanat KA, Bream KD, Holmes JH, Koenig HC, et al. Impact of store-and-forward (SAF) teledermatology on outpatient dermatologic care: A prospective study in an underserved urban primary care setting. J Am Acad Dermatol 2016;74:484-490.

50. Nami N, Massone C, Rubegni P, Cevenini G, Fimiani M, Hofmann-Wellenhof R. Concordance and time estimation of store-and-forward mobile teledermatology compared to classical face-to-face consultation. Acta Derm Venereol 2015;95:35-39.

51. Scheibe MM, Imboden JB, Schmajuk G, Margaretten M, Graf JD, Chen AH, et al. Efficiency gains for rheumatology consultation using a novel electronic referral system in a safety-net health setting. Arthrit Care Res 2015;67:1158-1163.

52. Fort MP, Namba LM, Dutcher $S$, Copeland T, Bermingham N, Fellenz $C$, et al. Implementation and evaluation of the safety net specialty care program in the Denver Metropolitan area. Permanente J 2017;21:16-22.

53. Liddy C, Singh J, Kelly R, Dahrouge $S$, Taljaard M, Younger J. What is the impact of primary care model type on specialist referral rates? A cross-sectional study. BMC Fam Pract 2014;15:22.

54. Jaakkimainen L, Glazier R, Barnsley J, Salkeld E, Lu H, Tu K. Waiting to see the specialist: Patient and provider characteristics of wait times from primary to specialty care. BMC Fam Pract 2014;15:16.

55. Joschko J, Liddy C, Moroz I, Reiche M, Crowe L, Afkham A, et al. Just a click away: Exploring patients' perspectives on receiving care through the Champlain BASE ${ }^{\mathrm{TM}}$ eConsult service. Fam Pract 2018;35:93-98.

Address correspondence to: Clare Liddy, MD, MSc, CCFP, FCFP C.T. Lamont Primary Health Care Research Centre Bruyere Research Institute 43 Bruyère St. Annex E, Room 106 Ottawa, ON K1N $5 \mathrm{C} 8$ Canada

E-mail: cliddy@bruyere.org

Received: January 5, 2018

Revised: March 21, 2018

Accepted: March 23, 2018

Online Publication Date: June 21, 2018 